\title{
Towards Integrating Traffic and Terrain Constraints into a Vertical Situation Display
}

\author{
P. Rijneveld*, C. Borst ${ }^{\dagger}$, M. Mulder ${ }^{\ddagger}$, M. M. van Paassen ${ }^{\S}$ \\ Delft University of Technology, Faculty of Aerospace Engineering Delft, The Netherlands
}

\begin{abstract}
Future airspace operations will allow flight crews to plan and fly their own preferred route and time of arrival without much intervention from air traffic control. This implies that pilots become more responsible for planning their own route while maintaining safe separation. This requires a strategic planning tool that support a high level of pilot Situation Awareness. Current aircraft are already equipped with terrain and traffic warning systems to, but these systems only provide short-term conflict resolutions. Recent studies have shown that a constraint-based approach to interface design could be used to support long- and medium-term decision-making and to provide a deeper understanding of the work domain. However, these studies were focused on either terrain or traffic. This paper aims to integrate both by showing the performance, traffic and terrain overlays on an experimental Vertical Situation Display. In a simulator evaluation, in which twelve professional airline pilots participated, this Vertical Situation Display was compared to a baseline Vertical Situation Display that only showed a terrain overlay and intruder aircraft relative to own ship. The experiment results revealed that the performance, traffic and terrain overlays improved pilots' overall situation awareness and pilots rated their workload lower. Regarding decision-making, however, the results were not significantly better. Regarding safety, there were less traffic conflicts, but more ground proximity intrusions.
\end{abstract}

\section{Introduction}

$T^{\mathrm{O}}$ meet the future demand of air transport, to increase the safety of flight and to decrease its environmental 1 impact, airspace operations are changing. New concepts for Air Traffic Management, such as SESAR, permit a more flexible use of airspace. ${ }^{1}$ Air traffic operations will be based on trajectory optimization instead of procedural control and the flight crew will have more responsibility to maintain safe separations. To enable pilots to monitor their designated trajectories, interfaces are required that support pilots in medium- and long-term strategic planning. Aircraft are already equipped with modern systems for detecting safety threats, such as other aircraft (ACAS), ${ }^{2}$ terrain (TAWS), ${ }^{3}$ a combination of both $\left(\mathrm{T}^{2} \mathrm{CAS}\right)^{4}$ and bad weather (weather radar). However, these systems have also a number of drawbacks. First, they are designed for short-term conflict avoidance and are only activated when hazardous situations are already at hand, and then require immediate and correct actions of the pilot. Second, as a result of sudden alerts the stress level of pilots increases significantly in situations that might already be dangerous. Third, these systems suffer from issuing false alarms which can cause a decrease of trust in the system. ${ }^{5}$ By hiding the rationale behind the commands, pilots may not be able to understand the reason for the alarms. ${ }^{6}$ Fourth, the use of explicit commands does not allow pilots to explore other solutions than those commanded by the automation. Additionally, the majority of aircraft accidents are the consequence of multiple failures in a setting that was not foreseen by the system designers and the automation. Unless there is a method to foresee all these

*MSc Student, Control and Simulation Division, Faculty of Aerospace Engineering, Delft University of Technology, Kluyverweg 1, 2629 HS Delft, The Netherlands. E-mail: p.rijneveld@gmail.com

${ }^{\dagger}$ Research Associate, Control and Simulation Division, Faculty of Aerospace Engineering, Delft University of Technology, Kluyverweg 1, 2629 HS Delft, The Netherlands. E-mail: c.borst@tudelft.nl

${ }^{\ddagger}$ Professor, Control and Simulation Division, Faculty of Aerospace Engineering, Delft University of Technology, Kluyverweg 1, 2629 HS Delft, The Netherlands. Senior Member AIAA. E-mail: m.mulder@tudelft.nl

$\S$ Associate Professor, Control and Simulation Division, Faculty of Aerospace Engineering, Delft University of Technology, Kluyverweg 1, 2629 HS Delft, The Netherlands. Member AIAA. E-mail: m.m.vanpaassen@tudelft.nl 
currently unforeseeable accidents and incidents, pilots must be prepared for handling these situations. This can be done by training and by providing the proper "tools" on the flight deck.

Since more separation assurance tasks are expected to shift from Air Traffic Control (ATC) towards the flight deck, it is important that a strategic planning tool shifts the short-term collision avoidance tasks towards medium- and long-term separation assurance strategies. Although pilots may prefer to delegate such tasks to the automation, and may even be dependent on its proper functioning, we feel that they should not be restricted in applying their expertise to control and guide their aircraft. Instead of focusing on developing more advanced and intelligent automated agents, a human-centered approach considers the ideal outcome of flight deck automation as a cooperative process, where the automation enables pilots to function to their full potential as creative problem solvers and decision makers.

Previous research has shown that using a flight display to present the relation between the 'internal' and 'external' constraints to flight can be an effective way to promote pilot SA and support decision-making. ${ }^{7-12}$ Previous research on increasing pilot SA through a Vertical Situation Display (VSD) focused on either visualizing traffic or terrain constraints. ${ }^{13,14}$ The goal of this paper is to combine these constraints on a new experimental VSD (EVSD) that can be used for long- and medium-term trajectory planning and separation assurance in the vertical plane.

The structure of this paper is as follows. First, a short overview is presented of previously designed interfaces meant to increase SA in the vertical plane and their experiment results. The third section provides the basic design of the new EVSD interface and a description of the interface content. The last part of the paper contains the description and the results of a pilot-in-the-loop experimental evaluation.

\section{Review of earlier VSD enhancements}

Several conceptual studies on cockpit interface design formed the basis for this research. Two of these studies, which were on enhancing the VSD, will be briefly reviewed.

\section{A. The Vertical Separation Assistance Display (VSAD)}

The VSAD was meant to provide the pilot a tool to maintain separation with respect to other aircraft in the vertical plane, ${ }^{13}$ see Figure 1.

\section{Constraints}

The internal constraints were formed by the performance envelope of the aircraft. This was an area described by the maneuver bounds for stall speed, maximum speed, maximum thrust and idle thrust. The minimum speed is defined by the stall characteristics, the maximum speed by the structural limitations, and the

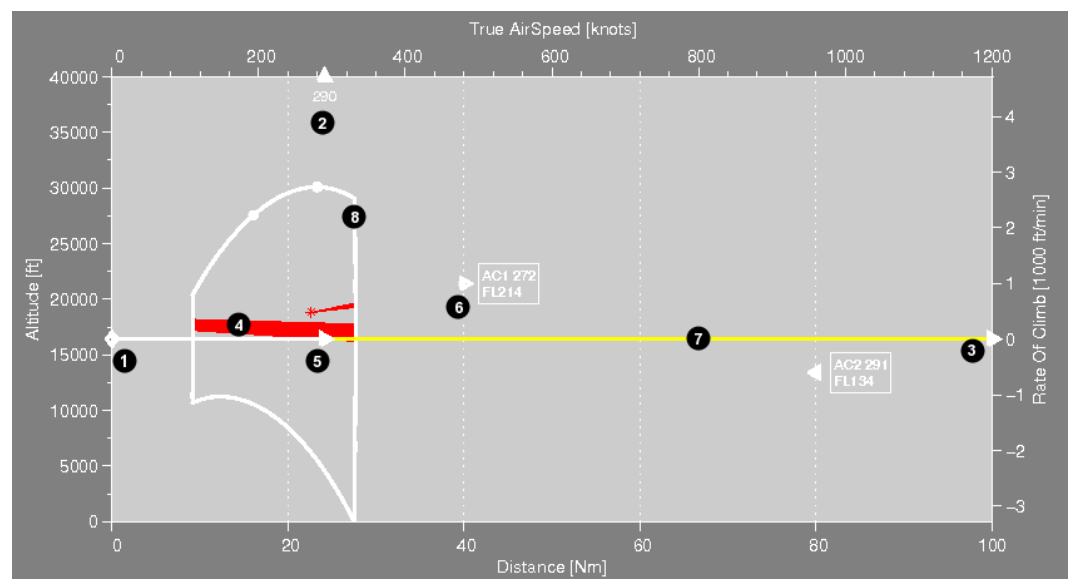

Figure 1. The VSAD. In this figure, (1) is the own aircraft symbol, (2) is the speed indicator, (3) is the vertical speed indicator, (4) is the conflict geometry overlay, (5) is the own speed vector, (6) shows the intruder aircraft with a flight label, (7) shows the own aircraft programmed flight path and (8) is the performance envelope overlay, transformed to the five minutes time interval. 


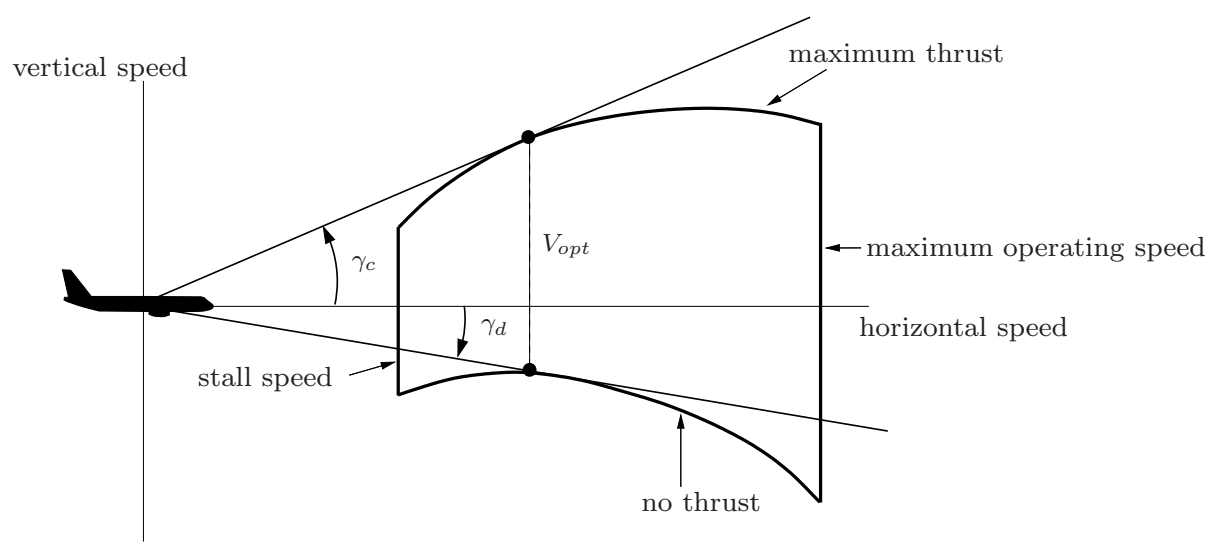

Figure 2. The performance envelope, where $V_{o p t}$ denotes the ideal climb and glide speeds, $\gamma_{c}$ the steepest climb angle, and $\gamma_{d}$ the optimum glide angle.

climbing capabilities by the maximum thrust of the engines. Also the gliding capabilities without thrust may be interesting, for example when an engine failure has occurred. Altogether these constraints form a boundary for stationary flight conditions, called the performance envelope, see Figure 2. As long as the flight path vector was kept within this area, the aircraft could obtain a stationary flight condition.

Minimum safe separation with respect to traffic can be defined using a virtual coin-shaped area around each aircraft, known as the Protected Zone (PZ). The dimensions of this area are the current separation minima: a height of 1,000 ft above and below the aircraft and a range of $5 \mathrm{NM}$, see Figure 3(a). When an aircraft enters the PZ of another aircraft, the separation criteria are violated, see Figure 3.

On the basis of the flight path vectors of both aircraft in the vertical plane a conflict geometry can be defined that indicates whether or not the aircraft are on collision course. Figure 4 indicates how this is done: from the ownship position two lines are drawn to the most left and most right point of the intruder aircraft. This is called the Forbidden Beam Zone (FBZ). As long the relative flight path vector stays outside of the FBZ, there will be no loss of separation. However, steering the relative flight path vector is a rather difficult and not very intuitive task. By applying basic vector geometry the FBZ can be translated in the absolute plane such that pilots only have to steer their own flight path vector out of the FBZ.

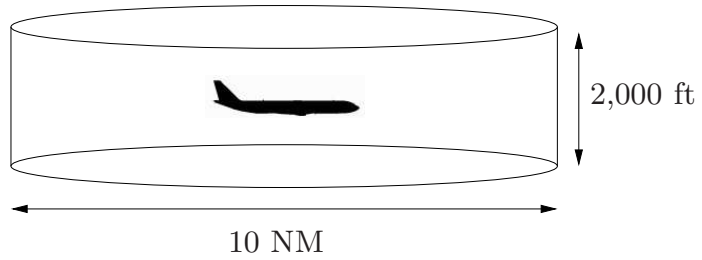

(a) Definition of the Protected Zone

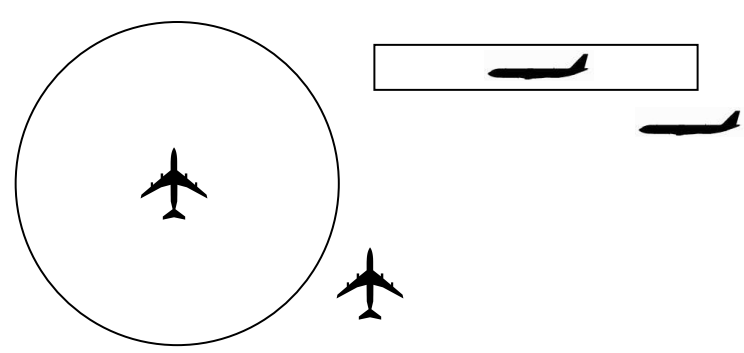

(b) Traffic separation criteria are not violated.

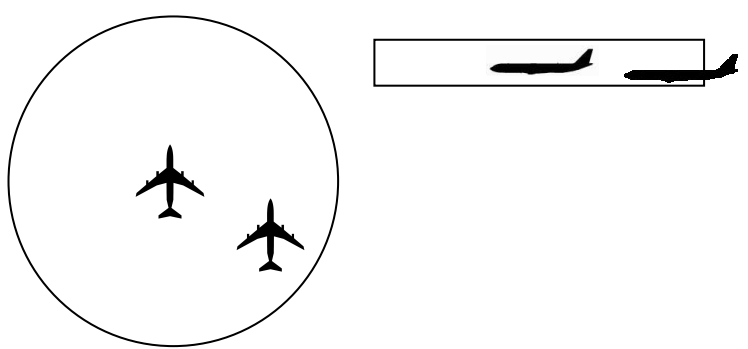

(c) Traffic separation criteria are violated.

Figure 3. Definition of the separation criteria. 


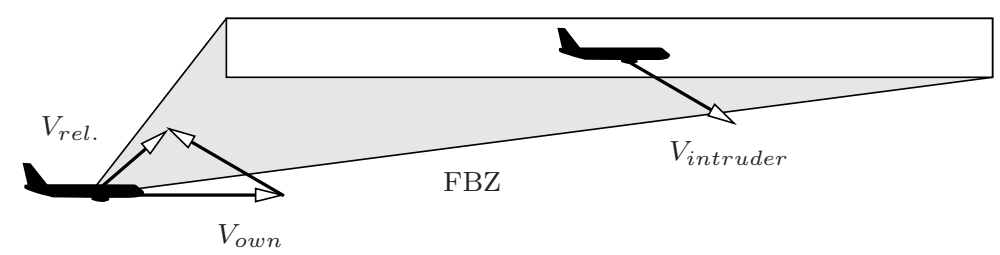

(a) The FBZ in the relative plane.

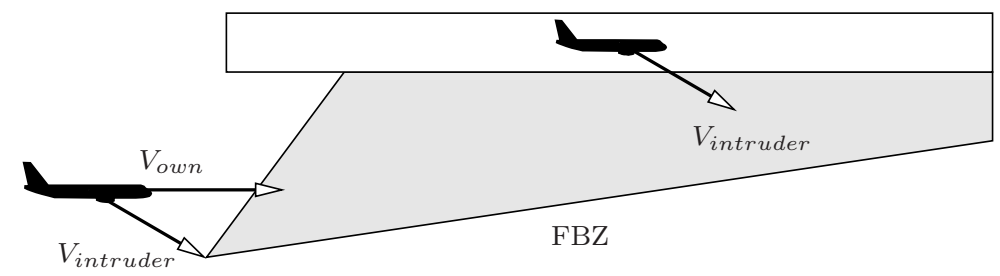

(b) The FBZ in the absolute plane.

Figure 4. Definition of the Forbidden Beam Zone (FBZ) in the relative and absolute velocity planes.

\section{Mapping}

Besides the altitude and distance tapes, the display (see Figure 1) also contained a speed and a vertical speed tape. The reason for showing these tapes was that the performance envelope and the FBZ are based on speed vectors. The mapping of the axes was consistent, meaning that the flight path vector indicated both the airspeed as well as the distance covered within a certain time interval (which was five minutes). The concept of the FBZ can be combined with the performance envelope: the FBZ then marks the area within the performance envelope where the tip of the flight path vector may not be located. To maintain separation, pilots should keep their flight path vector out of the FBZ to avoid a conflict and within the performance envelope to maintain a safe flight condition.

\section{Experiment results}

From an off-line experimental evaluation it was concluded that the pilots' traffic awareness improved considerably compared to a traditional VSD. Pilots indicated that they better understood when they were in conflict, could better locate the intruder aircraft, and were better able to determine an appropriate resolution maneuver, not only to avoid the conflict, but also to prevent other conflicts from occurring. The visualization of the performance and traffic constraints proved to be a useful tool to determine the resolution maneuver for the current conflict situation. It was further shown that increasing the number of intruder aircraft did not lower pilot subjective traffic SA. However, there was a low confidence level of the answers to the SA questions. This might be because the experiment was not a pilot-in-the-loop experiment, as the subjects had to watch videos showing the display behavior in conflict situations.

It was recommended to conduct a pilot-in-the experiment in which pilots should be actively involved in solving traffic separation conflicts. Regarding the display design, it was suggested to better visualize the connection between the FBZ and the intruder aircraft to which it belongs. Furthermore, a color coding scheme should be added to the FBZs indicating the urgency of the conflict. Also, there was a need to show the area behind the own aircraft, although conflict information about aircraft located behind was shown on the display. It was also suggested to investigate what the best resolution maneuvers are in terms of efficiency and safety.

\section{B. The Performance-based Vertical Situation Display (PVSD)}

The PVSD shows the maximum and minimum vertical maneuver capabilities of the aircraft, thereby providing the pilot the possibilities of climbing over terrain, ${ }^{14}$ see Figure 5. 


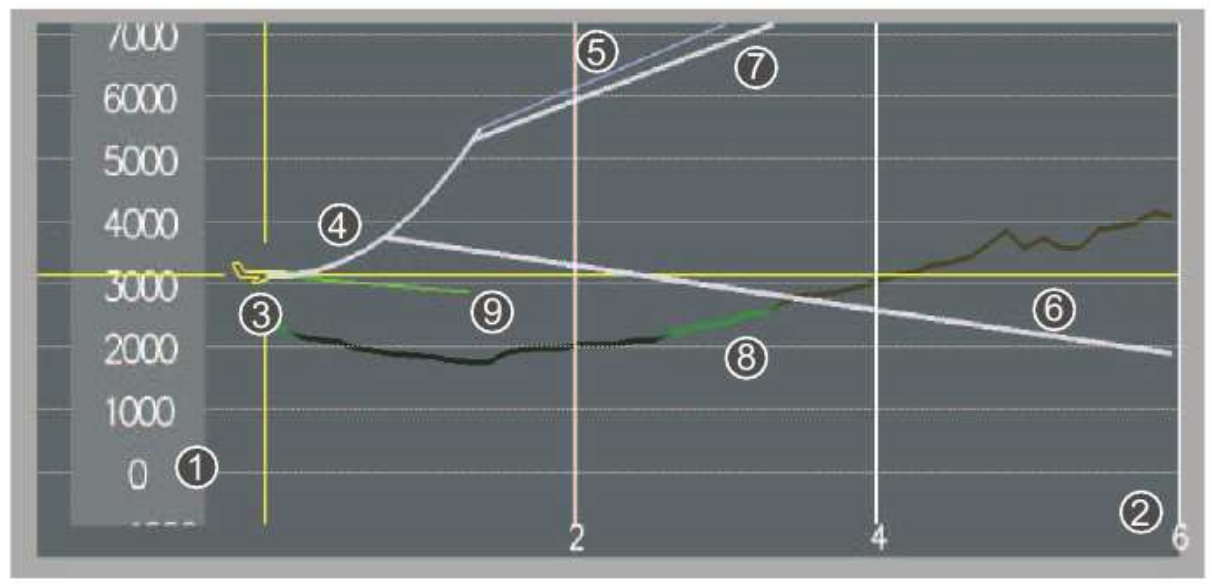

Figure 5. The Performance-based VSD. In this figure, (1) is the altitude tape, (2) is the look-ahead distance, (3) is the aircraft symbol, (4) represents the pull-up maneuver to obtain both the optimum climbing flight (5) and the optimum gliding flight (6); (7) represents the climbing limitations with climb power, (8) indicates the terrain in the vicinity of the aircraft, along the current flight path (9), which is predicted for 30 seconds ahead.

\section{Constraints}

In the vertical plane three types of dynamic maneuver boundaries are important: the pull-up/push-over maneuver, the optimum quasi-stationary climbing flight and the optimum gliding flight in case of total engine failure, see Figure 6. A pull-up/push-over maneuver is used to exchange kinetic and potential energy. The optimum climb is defined as the steepest climb with maximum (climb) thrust and maximum lift-to-drag ratio. The optimum glide is defined as the maximum range gliding flight without thrust, also with maximum lift-to-drag ratio.

An important factor is energy management: total energy is added to the system by means of the thrust lever and limits the climb performance of the aircraft, while the difference between the optimum airspeed and the current airspeed is a measure for kinetic energy that can be transformed into potential energy or vice versa.

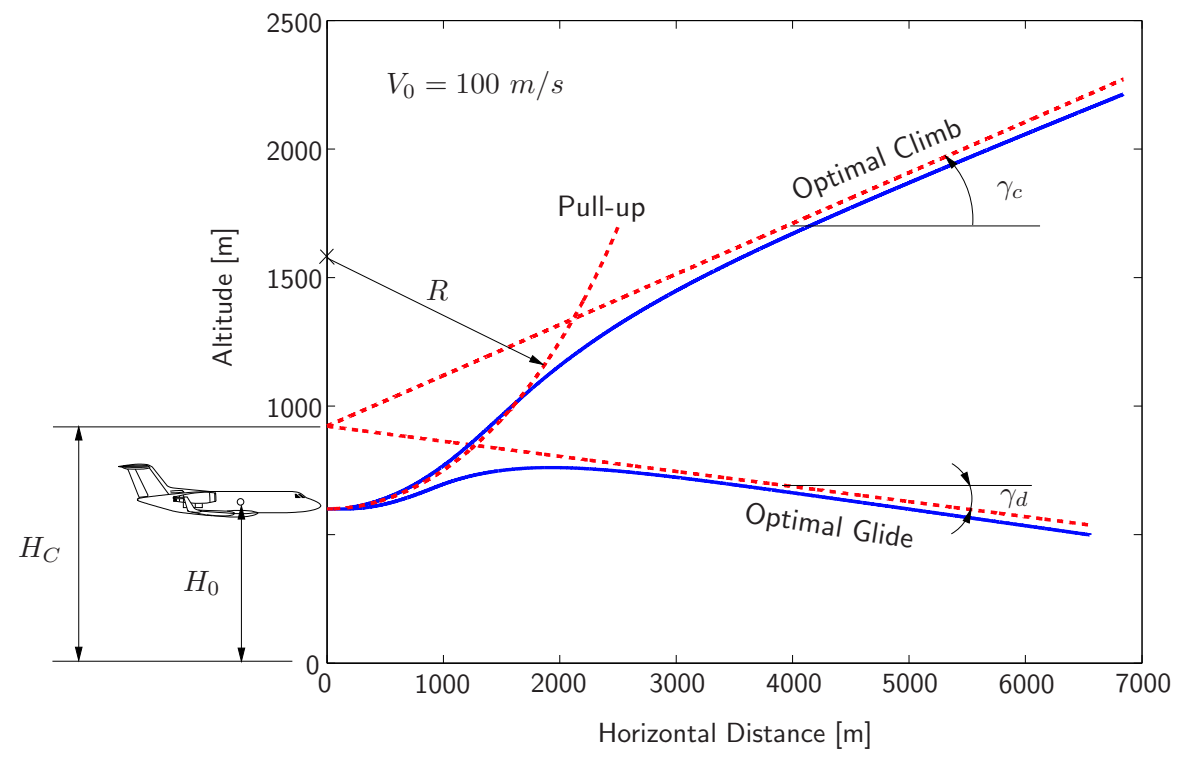

Figure 6. The optimum climbing and gliding flight. 


\section{Mapping}

The maneuver boundaries are projected onto the VSD, as shown in Figure 5. As long as the optimum climbing flight line is kept above the terrain obstacles, the aircraft possesses enough energy to climb over the terrain. Besides, if the terrain obstacles are also kept below the optimum gliding flight line, the aircraft also has enough energy to climb over the terrain in case of a (total) engine failure.

\section{Experiment results}

The results of the pilot-in-the-loop experimental evaluation showed that the design of the PVSD improved the pilots' spatial and risk awareness and reduced the pilot workload. Surprisingly, from the safety measurements it became clear that the PVSD resulted in a relatively large number of intrusions, where an intrusion was defined as entering a safe zone above terrain. Apparently, pilots accepted intrusions because they felt safer with the PVSD. Despite the number of intrusions with the PVSD, the number of crashes was much smaller. It was observed that the crashes occurred in the scenarios where there was an engine malfunction. It was hypothesized that, although the interface showed the limit performance characteristics, adding explicit energy management cues could support pilots in better executing the optimum climbing and gliding flight, and other strategies in between.

It was also observed that when not showing the internal aircraft and external terrain constraints, pilots adopted a strategy to be very cautious and reacted immediately when they felt insecure. Hence, the maneuver information on the PVSD could be an effective tool to detect and prevent a possible threat to safety. It was recommended to optimize the prediction algorithm for the transition between the pull-up maneuver and the quasi-steady climbing and gliding flight path.

\section{Combined Interface Design}

Mapping all constraints into one display is the challenge of this research. One of the major difficulties in mapping the constraints is that they apply to different domains: traffic constraints are mostly defined in the speed domain while terrain constraints are defined in the distance domain. Also the choice for a display range may impose problems, since traffic conflicts normally require a different look-ahead time than terrain conflicts.

\section{A. Integrating speed and distance domains}

The experimental VSD (EVSD) has four axes: a speed tape, a vertical speed tape, an altitude tape and a distance tape, see Figure 7. The altitude tape and distance tape are standard in the VSD and allow a physical representation of the objects in the outside world. The speed tape and vertical speed tape are needed to add the maneuver limitations in the form of the performance envelope and the limitations due to other aircraft. The relation between the speed tape and the distance tape is the look-ahead time. Therefore, the flight path vector not only indicates the airspeed (length) and flight path angle (direction), but also the horizontal distance and altitude that can be reached within the look-ahead time.

\section{The performance envelope}

Since the performance envelope is dependent (amongst others) on altitude, mapping it directly onto the distance axes does not result in a consistent mapping. Regarding the maximum thrust boundary, showing this boundary in the distance domain results in a downward shift of the maximum climbing capabilities in the speed domain, which in its turn results in another downward shift of the maximum thrust line in the distance domain. As a matter of fact, from simulations it was concluded that the discrepancy due to not correcting for altitude is small enough to neglect. However, it must be stressed that showing the performance envelope in speed terms without correcting for altitude results in the visualization of slightly over- or underestimated achievable distances in the distance domain.

The traffic constraints are visible inside the performance envelope. As long as the pilot keeps the tip of the flight path vector out of the FBZ, there is no loss of separation. Since the shape of the FBZ does not provide a direct cue on the distance- and time-of-conflict a color coding is added to the FBZ: it colors yellow when the intruder is more than 2.5 minutes away, amber when the intruder is between 1.5 and 2.5 minutes away and red if the intruder is closer than 1.5 minutes. 


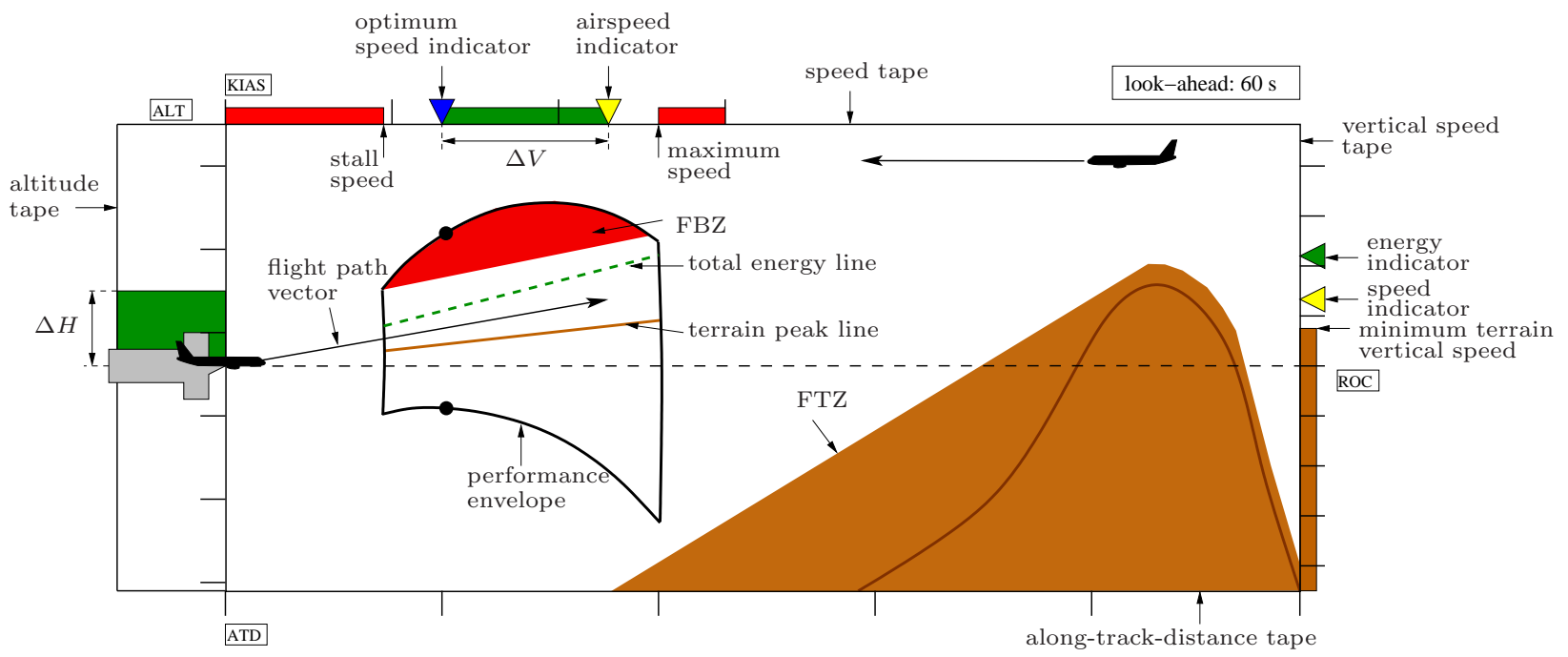

Figure 7. The constraints mapped on the EVSD.

\section{The speed tape}

For a consistent mapping of the speed and distance domains, the speed tape should actually represent the horizontal airspeed. However, normally the speed tape (on the PFD) represents the total airspeed. Mapping the total airspeed means that lines of constant airspeed become slightly curved, as can bee seen in Figure 8. This difference is proportional to the cosine of the flight path angle:

$$
V_{\text {offset }}=\frac{V}{V \cos \gamma}=\frac{1}{\cos \gamma}
$$

As a result the place of the speed indicator on the speed tape is also function of the flight path angle, which means that the speed tape must be scaled depending on the flight path angle. Since this is unwanted, a small discrepancy remains which is indicated in Figure 8.

Also, a consistent mapping of the airspeed requires that the true airspeed is shown, but normally the speed tape presents the indicated airspeed. In order to display the indicated airspeed, the speed tape must be scaled according to the difference between true airspeed and indicated airspeed.

The speed tape further indicates the minimum and maximum speed boundaries, the optimum speed for climbing and gliding, the current speed and the difference between the optimum speed and the current speed. This is the excess (or deficiency) of kinetic energy.

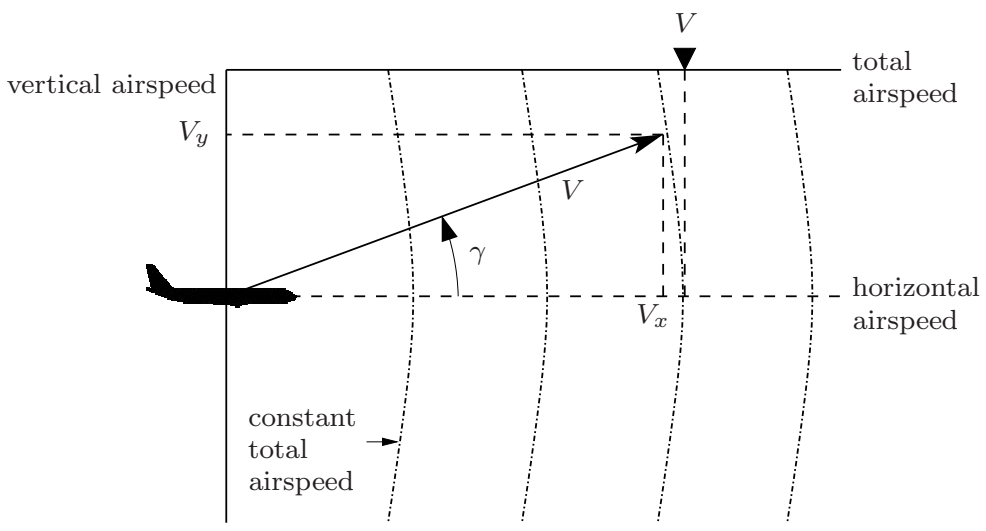

Figure 8. The speed tape offset. 


\section{The altitude tape}

The altitude tape indicates the current altitude and the excess (or deficiency) of potential energy. This is the same altitude difference as indicated in Figure 6. So from the speed and altitude tape the pilot can see the amount of kinetic energy that can be exchanged for potential energy or vice versa.

\section{The zoom function}

The pilot can change the range of the display manually. When the range is adjusted, the position of the physical objects change as well as the scaling of the distance tape and altitude tape. Changing both tapes keeps the aspect ratio of the display constant, which means that all angles stay constant. Another option would be to scale the speed tape and vertical speed tape, but the advantage of scaling the distance and altitude tape is that this is the natural way of scaling a display. Also, by not scaling the speed tapes the performance envelope stays constant (apart from altitude changes), although the boundaries will represent different distances when changing the range. The relation between the speed and distance domains, the look-ahead time, is shown in the upper-right corner of the display.

\section{The total energy line}

The total energy line is shown inside the performance envelope.

\section{The terrain peak line and the Forbidden Terrain Zone}

The highest point of the terrain within a certain scan range is indicated by the terrain peak line. Pilots are allowed to place the flight path vector underneath the terrain peak line, as long as the aircraft (symbol) stays out of the Forbidden Terrain Zone (FTZ), which is a projection of the climbing limitations of the aircraft onto the highest terrain peaks. A Minimum Safe Altitude (MSA) is included into the FTZ.

\section{The vertical speed tape}

The vertical speed tape is used to show the differences between the own vertical speed, the energy rate and the minimum vertical speed to avoid terrain.

\section{B. The extended performance envelope}

The performance envelope forms the boundary for the maneuver constraints, but it comprises only the stationary part of the total flight envelope. It is well possible to fly above the maximum thrust line or below the no-thrust line. However, then the airspeed will decrease or increase, respectively. So this is not favorable situation when solving a conflict. Only the minimum and maximum speed boundaries are fixed. When an energy exchange maneuver is executed it might even be unavoidable to fly outside the boundaries, because the pilot needs to pull-up the aircraft to a flight path angle that is larger than the optimum climb angle. Therefore, the external constraints are also shown above and below the maximum thrust and glide line, respectively. The minimum and maximum speed boundaries are extended by dashed lines. A screen capture of the final design of the EVSD is shown in Figure 9.

\section{Working with the cues}

Figure 9 shows an example situation of conflict solving with the EVSD. The constraint overlays are continuously presented, even when there is no immediate threat to safety. As such, pilots can early detect possible threats to safety and avoid them and are able to choose an efficient and economic maneuver.

\section{A. Traffic avoidance}

When an intruder aircraft comes within a predefined distance and altitude range of the own aircraft, the FBZ of this aircraft becomes visible on the EVSD. Figure 10 shows what the conflict geometry looks like when two aircraft are flying in different directions. From the shape of the FBZ it might be difficult to obtain a notion of the flight direction of the intruder and the severity of the conflict. However, the FBZ has some properties that might be useful. 


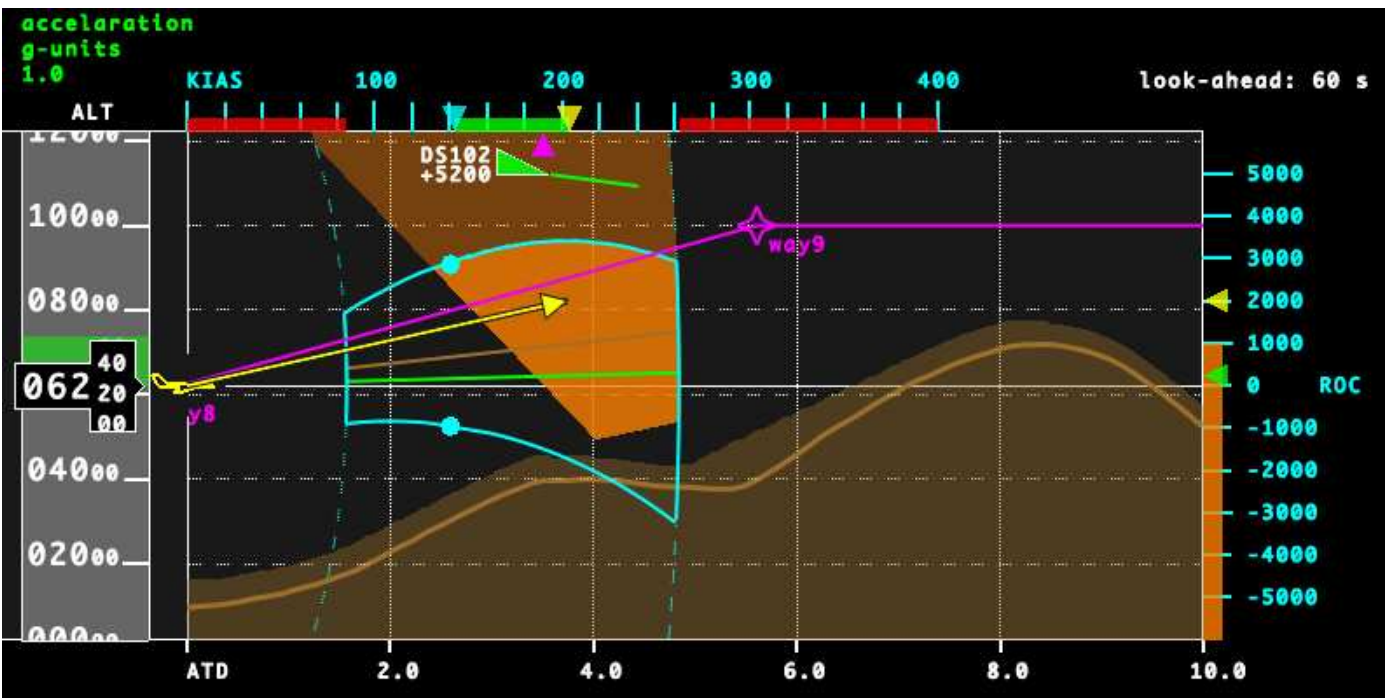

Figure 9. The EVSD interface.

- The angle between the legs of the FBZ gives pilots an idea of the distance of the intruder aircraft. A large angle between the legs indicates that a conflict is close, while a small angle indicates that the intruder aircraft is far away.

- The tip of the FBZ represents the flight path vector of the intruder aircraft, see Figure 10(a). So when this tip is visible, the flight direction and airspeed of the intruder aircraft can be derived.

- When this tip is visible, the intruder aircraft flies in the same direction as the own aircraft. When the intruder aircraft is flying in the opposite direction, the tip of the FBZ is certainly not visible.

- When avoiding a traffic conflict, the tip of the flight path vector of the intruder aircraft has to be avoided, since this means that the two aircraft are flying exactly parallel and the conflict will not be solved.

- If the own flight path vector is outside the conflict area, which indicates a safe situation, the traffic conflict area will develop in a direction away from the own flight path vector, so the situation becomes even more safe. On the other hand, when the flight path vector is inside the conflict area, the conflict area will expand around the own flight path vector, making the conflict more severe.

When the conflict zone is not yet colored red, pilots have some time to move their flight path vector in and out of the conflict area to search for the best resolution. When the conflict zone turns red, a short-term conflict avoidance maneuver must be executed: pilots should take immediate action to move the flight path

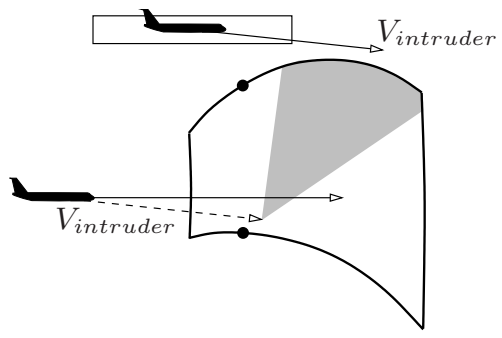

(a) The traffic conflict geometry when the aircraft fly parallel.
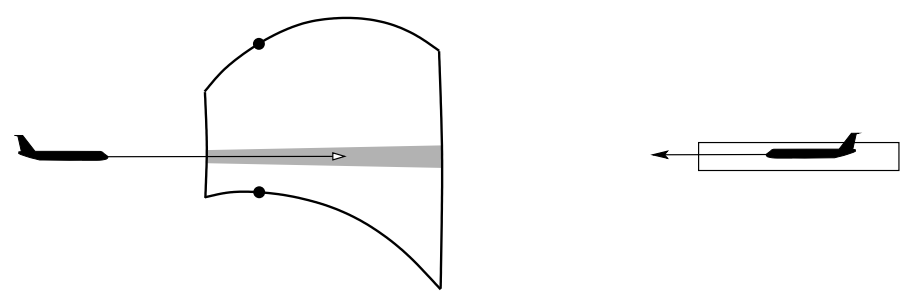

(b) The traffic conflict geometry when the aircraft fly in opposite directions. Changing speed only will not result in resolution of the conflict.

Figure 10. Cues from the traffic conflict geometry. 
vector out of the FBZ. As a matter of fact, the solution space becomes smaller and smaller when the flight path vector is kept inside the FBZ.

Pilots have to be aware that the performance boundaries shift when the altitude is changed. Especially the maximum thrust boundary must be monitored when choosing a resolution in the upper part of the performance envelope, because when the aircraft is climbing the maximum thrust boundary will shift downwards, thereby reducing the solution space.

\section{B. Terrain avoidance}

Figure 11 presents some examples of the cues obtained from the energy angle, flight path vector and terrain peak line. For long-term terrain avoidance both the flight path vector and the energy line should be above the terrain peak line. Pilots can use the difference between the flight path angle and the energy angle as a measure of acceleration: when the energy line is higher than the flight path vector, the aircraft is accelerating; when the energy line is lower than the flight path vector, the aircraft is decelerating.

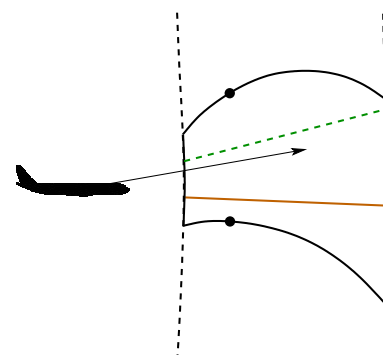

(a) A safe situation, where the flight path vector and energy line are above the terrain peak line. The aircraft is accelerating.

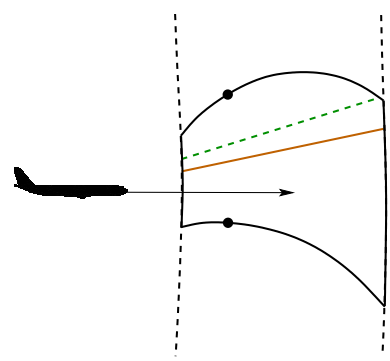

(e) An unsafe situation because the flight path vector is not aimed above the terrain. There is, however, enough energy in the system to climb over the terrain.

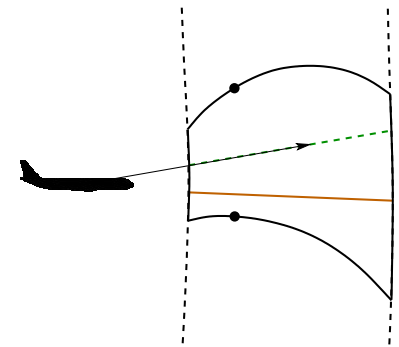

(b) Also a safe situation, the aircraft flies at constant airspeed above the terrain.

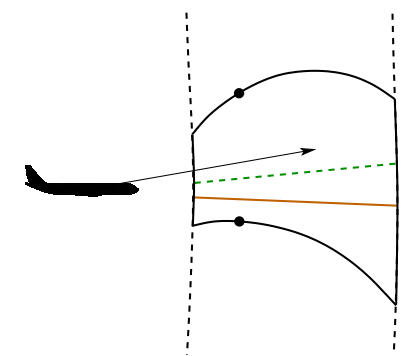

(c) A safe situation, but the aircraft is now decelerating.

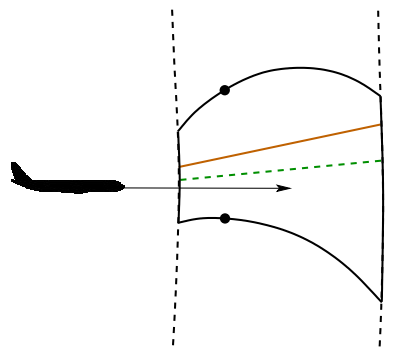

(d) An unsafe situation: the amount of energy and the flight path vector are too low to climb over the terrain.

Figure 11. Cues from the energy angle (green, dashed line), the flight path vector and the terrain peak line (brown, solid line).

\section{Experimental evaluation}

To evaluate the EVSD as a strategic planning and decision support tool, an experimental evaluation was done. The experiment was a pilot-in-the-loop experiment with mixed within- and between-subjects independent variables and was conducted in a fixed-base flight simulator. Lateral control of the aircraft was fixed, so only vertical maneuvers were possible. This was because the EVSD only provides information in the vertical plane. Since the display was designed to provide maneuver constraints imposed by traffic and terrain, scenarios were created that let pilots deal with low-level terrain flying while other aircraft obstruct the intended flight path. Pilots had to make strategic decisions to avoid traffic and terrain collisions. 


\section{A. Method}

\section{Subjects and instructions}

In the experiment a total number of 12 professional glass-cockpit airline pilots participated, with an average age of 37 years and an average experience of 4600 flight hours. The subjects were randomly assigned to one of two groups. Each group had a different display configuration.

The pilots were instructed to follow a set of waypoints and speed commands, shown on the displays. During a run, they had to avoid traffic and terrain by adjusting either their airspeed or altitude, or both. They were not given specific strategies to solve the conflicts, because the display is meant to support decisionmaking, rather than command it. Therefore, they were told to solve conflicts in a safe way, with minimum deviations from the intended flight path and airspeed. The trade-off between safety and flight path efficiency was to be made by the pilots.

\section{Independent variables}

The independent variables in the experiment were as follows:

1. Within-subjects variables. The experiment had one within-subjects variable: the conflict scenario (SCENE). The scenarios were divided into traffic conflict scenarios (4), terrain conflict scenarios (3) and mixed scenarios (4). One scenario was conflict free. SCENE had thus 12 levels.

2. Between-subjects variables. Between subjects the display configuration (DISP) varied. The EVSD was compared to an alternative ecological VSD. This display was based on the PVSD ${ }^{14}$ (Section II) which showed the climb and glide limitations. The display configuration had two levels: VSD and EVSD. Since the two displays were quite different and require extensive training, it was decided to let each group test only one display.

\section{Scenarios}

The scenarios contained crossing traffic, high terrain without overflying traffic, or high terrain with crossing traffic. Traffic was placed in all quadrants with respect to the own aircraft and was flying either the same course or the opposite course. The pilots had to choose a resolution which was, in their opinion, both safe and flight path efficient.

SCEnARIO 1-4 Traffic conflict. An intruder aircraft crosses the intended flight path from above and below in the opposite and parallel direction. In these scenarios a collision avoidance maneuver is needed.

SCEnARIO 5 Terrain conflict. The flight plan contains a faulty waypoint that is placed inside a mountain and requires an evasive climb maneuver to avoid a terrain crash.

Scenario 6-7 Terrain conflict. The same as scenario 5, however, in these scenarios also an intruder aircraft is present. The intruder limits the climbing possibilities of the own aircraft, but the flight path of the intruder does not obstruct the intended flight path.

SCEnARIO 8-11 Traffic and terrain conflict. In these scenarios a traffic collision avoidance maneuver is needed, while high terrain requires the own aircraft to climb. There are no faulty waypoints, so regarding terrain the flight plan could be followed.

SCEnARIo 12 No conflicts. An intruder aircraft is present, but it does not obstruct the intended flight path.

\section{Display configuration}

The alternative VSD presented limitations on aircraft performance in the form of the optimum climbing flight line and optimum gliding flight line, a terrain contour and a flight path vector. For this experiment the intruder symbol (with an indication of the altitude difference) was added. Also, the traffic constraint was added by means of the PZ, see Figure 12. In order to make a fair comparison between the two display configurations, some information from the EVSD was added to the alternative VSD, namely: the speed tape, 
the vertical speed tape (including the minimum terrain rate-of-climb), the look-ahead time, and the energy zones on the speed tape and the altitude tape. Since the EVSD showed also conflict information about aircraft that were not visible on the display (for example, when they were behind the own aircraft), the design alternative showed a flight label near the boundary of the display where the intruder aircraft could be expected.

Both display configurations had a Primary Flight Display (PFD) with a computer-generated outside view (a Synthetic Vision System (SVS)), a Navigation Display (ND) to provide a top view of the situation, and an engine display to show the engine status, see Figure 13.

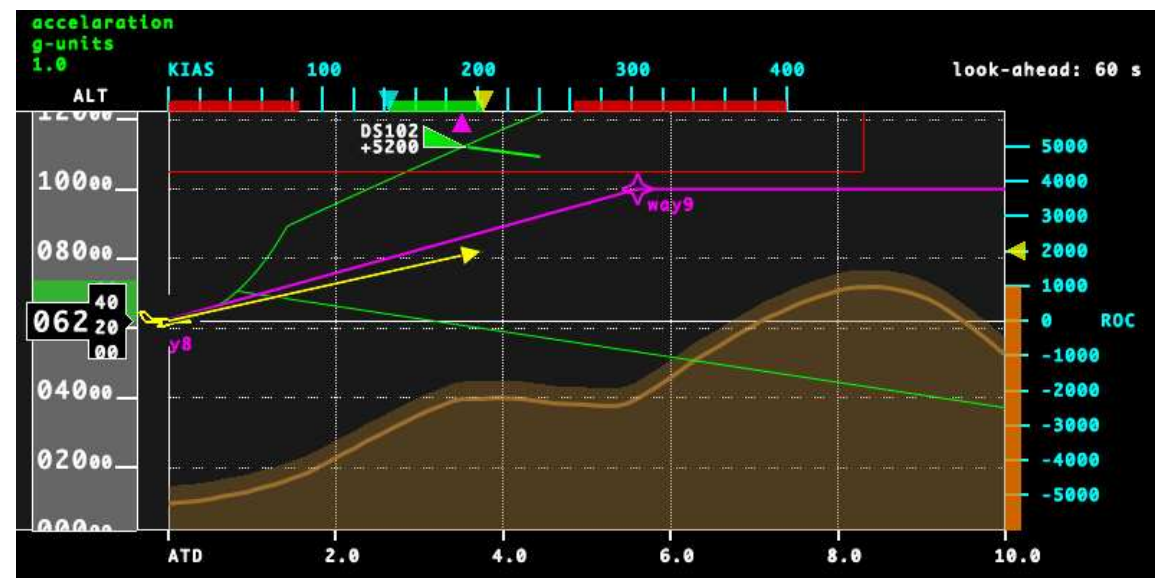

Figure 12. The alternative VSD.

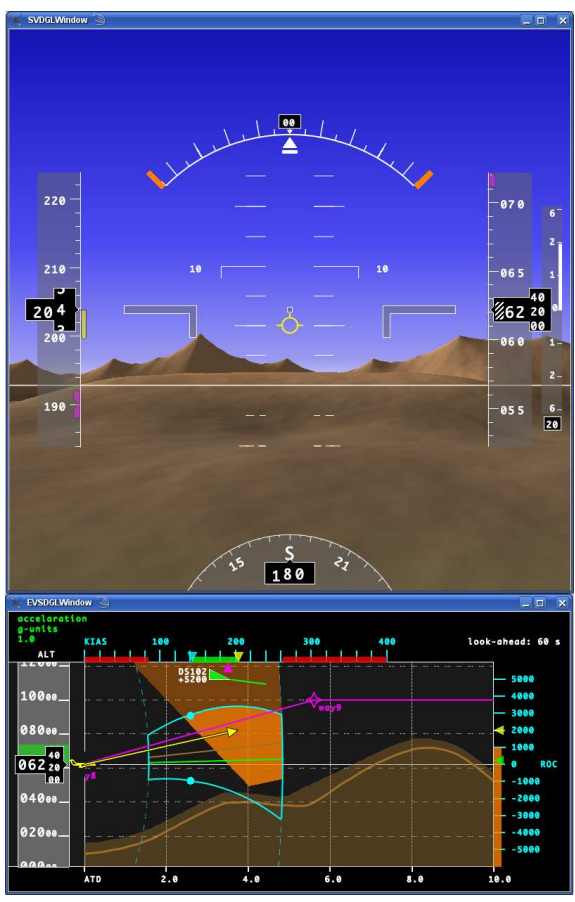

(a) The screen containing the SVS and the VSD/EVSD.

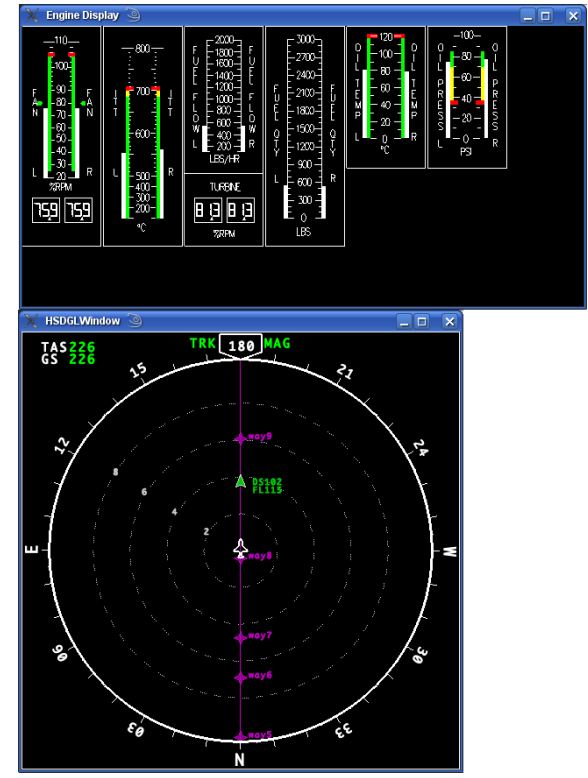

(b) The screen containing the Engine Display and the ND.

Figure 13. An overview of the experimental screens. 


\section{Apparatus}

The experiment was conducted in a fixed-base flight simulator, consisting of a cabin mockup. The cabin had two 18" LCD-monitors. One was situated in front of the pilot, which was used to display the SVS and VSD/EVSD. The other display was situated to the left of the pilot and showed the ND and the engine instruments. The aircraft was controlled by a control-loaded side stick on which fixed mass, spring and damper settings were selected. Furthermore, a throttle quadrant was used. The experiment was conducted head-down.

\section{Aircraft characteristics and experiment conditions}

The aircraft model used in the simulation is a six degrees of freedom non-linear Cessna Citation 500 model. ${ }^{15}$ In order to relieve the pilot from having to trim the aircraft all the time, a pitch rate controller was used, which worked as a pitch attitude hold mode. Lateral movements were fixed. The aircraft was trimmed at several altitudes and airspeeds as required for the scenarios. The atmosphere was modeled according to an International Standard Atmosphere without wind and without turbulence.

\section{Dependent measures}

The dependent measures in the experiment were:

1. Performance/decision. The pilot's performance was rated by grading the initial escape maneuver. Choosing the escape maneuver that is the most flight path efficient and also safe was rated 2. Criteria for an efficient, smooth maneuver were a maximum airspeed difference $\Delta V$ with respect to the commanded airspeed of $40 \mathrm{kts}$, a flight path angle $\gamma$ between -6 and 10 degrees and/or a maximum, instantaneous vertical load factor $n_{z}$ of $1.5 \mathrm{~g}$. Choosing the optimal escape maneuver but executing it less efficiently was rated 1 . A non-optimal initial escape maneuver was rated 0.

2. Situation awareness. SA was subjectively measured by a questionnaire when the pilots had finished the experiment. Additionally, traffic awareness was subjectively measured during the experiment by asking verbally at the start of each conflict situation: Do you think this intruder causes a traffic conflict? The answers were graded according to Table 1.

3. Workload. The workload was measured by means of subjective data from the NASA-TLX form, completed by the pilots after each run.

4. Safety. The safety with respect to terrain was measured by the number of crashes and the number of intrusions into the region between $0 \mathrm{ft}$ and $500 \mathrm{ft}$ above the terrain's surface. The safety with respect to traffic was measured by the number of intrusions into the $\mathrm{PZ}$ of the intruder.

Table 1. Grade determination of the inflight SA answers.

\begin{tabular}{ccc}
\hline \hline & \multicolumn{2}{c}{ Answer } \\
\cline { 2 - 3 } & Incorrect & Correct \\
\hline Sure & 0 & 3 \\
Unsure & 1 & 2 \\
\hline \hline
\end{tabular}

\section{Final questionnaire}

When the pilots had finished the experiment, they were asked to complete a final questionnaire that contained questions about the pilot's overall SA and the pilot's ability to perform the tasks with the display. Furthermore, the questionnaire contained questions about the symbology. 


\section{Experiment design and procedure}

The pilots were divided into two groups. Six pilots tested the EVSD while six other pilots tested the design alternative. After an explanation of the display features the pilots started with the training phase. In this phase they could practice with all 12 scenarios. They started with the no-conflict scenario, then they got the traffic conflict scenarios, followed by the terrain conflict scenarios and finally the mixed scenarios. After a small break, the measurement phase started, in which the subjects flew four long runs. Each run contained multiple conflict scenarios in a random order. Each conflict scenario was flown only once, resulting in 12 samples per pilot. Per group this resulted in $72(6 \times 12)$ samples.

\section{Results and discussion}

The analysis of the performance/decision and the inflight traffic awareness was done using a repeatedmeasures Analysis of Variance (ANOVA) with mixed within- and between-subjects variables. Since the dependent measures were expected to be correlated, a Multivariate ANOVA (MANOVA) was done. Pillai's trace tests were used because of the non-normal distribution of the data. ${ }^{16}$

No significant effect was found of DISP (Pillai: $F(2,9)=3.036, p>0.01$ ), a significant effect of SCENE (Pillai: $F(22,220)=2.367, p<0.01$ ) and no significant effect of the DISP $\times$ SCENE interaction (Pillai: $F(22,220)=1.442, p>0.01)$.

A non-parametric Spearman correlation test was done on the performance/decision and inflight traffic awareness measures, but no significant correlations were found.

\section{A. Performance/decision}

Analysis of the performance/decision measure showed that only SCENE had a significant effect on performance/decision $(F(11,110)=4.036, p<0.01)$. The average performance ratings for the scenarios are shown in Figure 14. The results are also grouped per conflict type, see Figure 15. Post-hoc analysis (SNK, $\alpha=0.05)$ did not reveal significant differences between these groups. From the figures it can be seen that in the traffic conflict scenarios the average scores were higher for EVSD, except for the first scenario. In this scenario the most efficient solution was to decrease speed in order to let the intruder aircraft pass. Also, the flight plan required the own aircraft to climb and in order to avoid the traffic, an even larger speed reduction was necessary. Some EVSD pilots thought they were safe after resolving the first part of the conflict by a small speed reduction, but they did not anticipate on the climb. The VSD pilots did not have a confirmation whatsoever whether a conflict was solved or not, so they had to monitor the situation more closely and this forced them to anticipate on the climb. In the second scenario a speed decrease was required. The EVSD showed this by coloring the right (high speed) part of the solution space. VSD pilots sometimes tried to overtake the other aircraft. The third scenario required a climb. It was observed that many VSD pilots performed an optimum climb, which was not the most efficient solution. To resolve the conflict in the fourth scenario, pilots could choose to either climb or descend (a descent, however, was slightly more efficient). A speed change did not solve the conflict, although some VSD pilots also performed a speed change. They indicated that they did this to resolve the conflict faster, which probably meant that they were unsure about the altitude change maneuver. Also in this scenario a number of optimum climb maneuvers was recorded. Although these maneuvers were the most safe, they were certainly not the most efficient.

Figures 14 and 15 show higher average scores for VSD for the terrain conflict scenarios, except for scenario 7. The differences in performance were caused by pilots operating with the EVSD who sometimes flew so close to the terrain, that they entered the terrain safety zone, resulting in a performance score penalty. This might indicate that the presented information allowed the pilots to fly on the safety limits.

For the mixed scenarios, the average scores were higher for EVSD, except for scenario 9. In scenario 8 the most safe and efficient resolution was to stay behind the intruder aircraft while climbing over the terrain. Hence, a speed increase was not the best solution. None of the EVSD pilots chose to increase speed, indicating that this was correctly shown on the display. A few VSD pilots however, did increase their speed. When scenario 9 was triggered, the vector geometry initially indicated a collision course because of a climbing intruder aircraft underneath the own aircraft. However, since the flight plan required the aircraft to climb anyway, this conflict could be easily solved. Because of the initial conflict prediction, some EVSD pilots reacted immediately by climbing. VSD pilots, which did not have conflict prediction, just followed their flight plan and stayed ahead of the intruder by small speed and/or altitude adjustments. In scenarios 10 and 


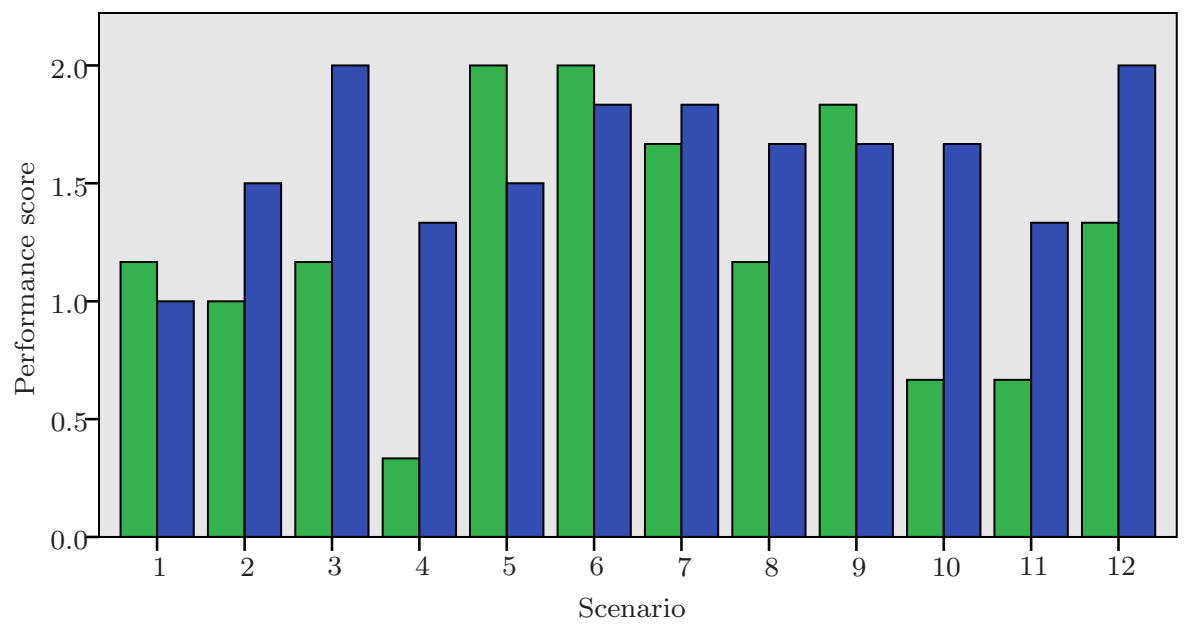

display $\square$ VSD

Figure 14. Average performance scores per scenario (scenario 1-4: traffic, 5-7: terrain, 8-11: mixed, 12: no conflict).

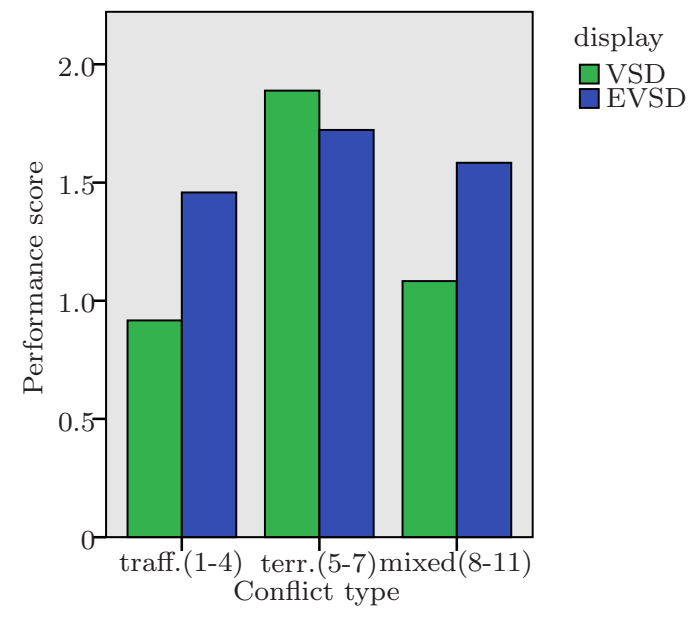

Figure 15. Average performance scores per conflict type.

11 pilots had to choose whether to fly between the mountain and the intruder aircraft or to climb over both. The first option was the most efficient. The EVSD pilots could see that flying between the obstacles was a safe option. The VSD pilots had to extrapolate the state information and were therefore not so confident. This resulted in more maneuvers where pilots chose to climb over both obstacles. It was also observed that more VSD pilots increased their speed, probably to resolve the conflict faster (which might indicate a lack of confidence in the initial altitude change maneuver). For scenarios 10 and 11 also more wrong initial decisions were recorded for VSD. Scenario 12 required no intervention by the pilot at all, however, some VSD pilots chose to climb over the intruder to avoid future problems with terrain that was far ahead (and thus not an immediate issue).

\section{B. Inflight traffic awareness}

There were no significant effects of DISP $(F(1,10)=3.876, p>0.01)$, SCENE $(F(11,110)=0.985, p>0.01)$ and their interactions on the inflight traffic awareness. A boxplot of the results is shown in Figure 16(a). Analysis of the results revealed that the inflight traffic awareness was higher or equal for EVSD for all scenarios, except for scenario 6 . In this scenario an intruder was ahead and overflying but of no danger, even when the pilot had to climb to avoid terrain. However, for EVSD, when the pilot started to climb, the 
flight path vector might have entered the FBZ at the top of the performance envelope, indicating a possible (long-term) conflict.

Further, the results revealed that the differences between the answers of the VSD pilots and the EVSD pilots were largest for the mixed scenarios, indicating that in complex situations the traffic awareness of EVSD pilots was higher than for VSD pilots.

Figure 16(b) shows the percentage of cases were pilots gave the correct answer and were also confident about it. This number was higher for EVSD. This result was tested on significance, but only a borderline significant effect was found of DISP $(F(1,10)=6.983, p=0.025)$.

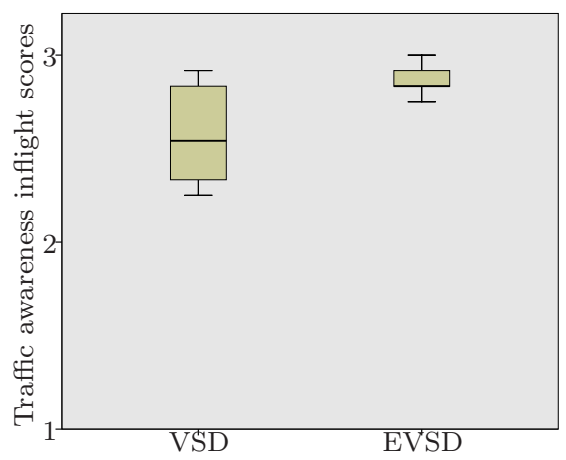

(a) Boxplot of the inflight traffic awareness answers

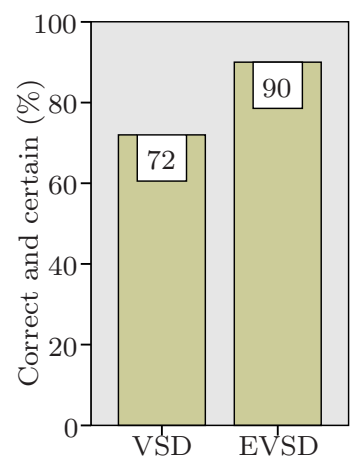

(b) Percentage of correct and certain answers

Figure 16. Boxplot of the inflight traffic awareness answers with the percentages of correct and certain answers.

\section{Workload}

Since workload was only measured at the end of each run and not for each individual scenario, a separate ANOVA (only for DISP) was done. This result was significant $(F(1,47)=11.542, p<0.01)$. Figure 17 shows a boxplot of the measurements, which indicates a higher workload for VSD. It must be noted that the total workload is difficult to compare for the two groups, because it was observed that both the magnitude and the duration of stressful situations varied between groups. EVSD pilots had to interpret more information at once when a conflict was triggered, but it might be that it was easier to evaluate whether a chosen strategy will remain conflict free. Hence, the EVSD pilots could have experienced a relatively high workload but only for a short amount of time. The opposite might be the case for VSD pilots. Investigation of the subscales of the query revealed that the most important factors 'mental demand' and 'temporal demand' were higher for VSD.

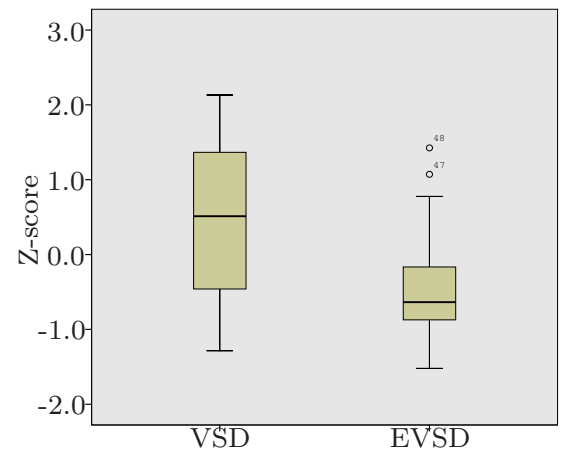

Figure 17. Boxplot of the workload measurements. 


\section{Safety}

The number of traffic PZ intrusions, terrain safety zone intrusions and terrain crashes are shown in Table 2. For EVSD all traffic intrusions occured in scenario 1. It was already discussed that pilots initially solved the conflict by decreasing speed, but they did not anticipate on the climb, where they needed an additional speed decrease. For VSD there was one traffic intrusion for scenario 1, but there were also traffic intrusions for scenarios 8 and 11. These were due to wrong decisions. As discussed, there were several terrain safety zone intrusions for EVSD in scenarios 5, 6 and 7. This was because pilots tried to fly as close as possible to the terrain to stay as close as possible to the flight path. Probably, the presented information gave the pilots confidence in their performance because the safety intrusions did not result in crashes.

Table 2. Safety in terms of traffic Protected Zone intrusions, terrain safety zone intrusions and terrain crashes. Between brackets are the number of possible conflicts.

\begin{tabular}{lcc}
\hline \hline & VSD & EVSD \\
\cline { 2 - 3 } traffic PZ intrusions (66) & 5 & 3 \\
terrain safety zone intrusions (42) & 0 & 4 \\
terrain crashes (42) & 0 & 0 \\
\hline \hline
\end{tabular}

\section{E. Final questionnaire}

\section{Situation awareness}

The results of the SA questionnaire are shown in Figure 18, of which the questions can be found in Table 3. Pilots had to rate their answers on a 10-points Likert scale. Pilots flying with the VSD found it easier to estimate the position of the intruder aircraft. That seems logic, because on the VSD a flight label was shown at the border of the display to indicate the position of the intruder aircraft when it was not within range. This feature was not present on the EVSD, since this display already provided cues for traffic conflict avoidance. The questions regarding the understanding of the maneuver possibilities of the aircraft with respect to traffic and terrain, resulted in higher SA ratings for EVSD. Further, the pilots that operated with the EVSD found it easier to choose an escape maneuver and felt less time pressure. Also, EVSD pilots had a better risk awareness than the pilots flying with the VSD.

It was observed that the VSD pilots used the zoom function on average more than the EVSD pilots. This indicates that the EVSD pilots were more confident about the situation than the VSD pilots. Actually, the EVSD was designed to show the conflict information independent of the display range setting.

Pilots operating with the EVSD noticed that they used the EVSD as a primary flight instrument instead of the PFD. This is explained by the fact that all information necessary to perform their task was present on the EVSD (however, also on the VSD). It also indicates that the EVSD is indeed useful, although it is certainly not meant to replace the PFD. As a matter of fact, it could even be combined with a constraintbased Synthetic Vision Display. ${ }^{9,10}$

Finally, all pilots (from both groups) indicated that the VSD/EVSD improved their (vertical) SA.

\section{Symbology}

Both groups evaluated the symbols on their displays. Pilots had to indicate whether they understood the symbols and whether they did or did not use them. It was not specifically asked whether pilots found the symbols useful, however some pilots added such remarks.

The energy bars on the speed and altitude tapes were not used by many VSD and EVSD pilots. VSD pilots found the optimum climb line a helpful feature, although the scenarios did not require the use of the maximum climbing capabilities. They suggested to add cues for conflict solving in between the ultimate margins. This is exactly what the EVSD is meant to provide. Further, some VSD pilots did not use the information on the vertical speed tape $(33 \%)$.

All EVSD pilots found the energy line very helpful for estimating their energy state (especially regarding high terrain) and for aiming their flight path vector more precisely (due to the acceleration indication). They liked this ability in combination with the conflict geometry, allowing them to point the flight path vector easily in a safe direction and airspeed. The opinions about the performance envelope as a boundary for 


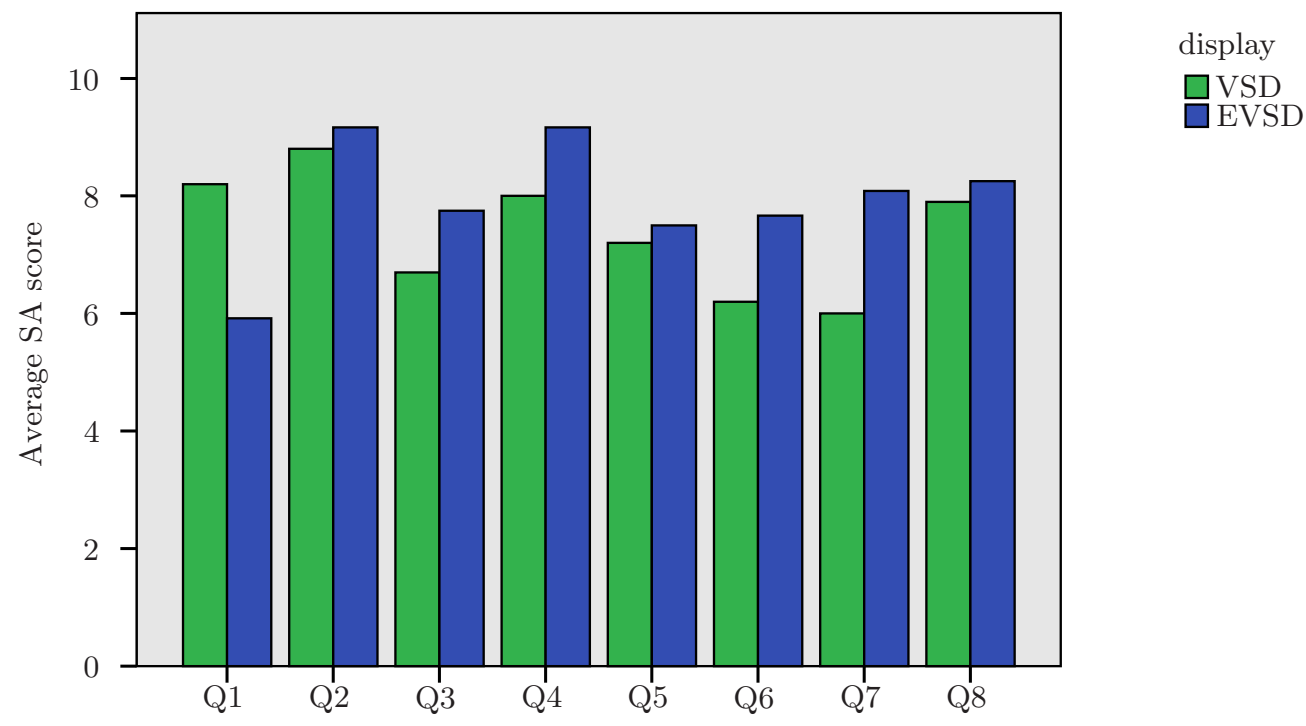

Figure 18. Results of the SA part of the final questionnaire.

Table 3. Questions of the SA part of the final questionnaire.

\begin{tabular}{l} 
Q1: How well were you able to estimate the position of the approaching intruder aircraft \\
Q2elative to your position? \\
Q3: How well were you able to estimate your position relative to the approaching terrain? \\
aircraft? \\
Q4: How well were you aware of the aircraft's capabilities with respect to avoiding terrain? \\
Q5: How would you rate the difficulty of following the trajectory with the given commanded \\
Q6: How would you rate the difficulty of choosing an escape maneuver? \\
Q7: How would you rate the amount of time available to solve the conflict? \\
Q8: Did you have a clear idea of the risk of the situations you experienced? \\
\hline \hline
\end{tabular}

showing the conflict geometry were mixed. One pilot liked it, while two others indicated that they did not need the performance boundaries to judge their performance capabilities and hence regarded them as clutter. However, the minimum and maximum speed boundaries were evaluated as helpful, although it was suggested to show more conservative boundaries (for example a minimum speed boundary representing $1.3 V_{\text {stall }}$ instead of $\left.V_{\text {stall }}\right)$. Some pilots (33\%) found the brown terrain peak line helpful, they even indicated that it should be more clear and on top of all other information. Regarding the range of the terrain scan (which was fixed at $15 \mathrm{NM}$ ), pilots indicated that that should be the same as the current display range. Pilots found the color coding of the traffic conflict geometry useful, although it was indicated that the color red should be preserved for real danger (50\%). When a traffic conflict is solved (meaning that the tip of the flight path vector is outside the FBZ), the zone should not turn red.

\section{Further pilot comments}

Pilots who tested the VSD found it difficult to estimate or predict the flight path of the intruder aircraft and relied very much on the textual information that was provided by the flight label. They indicated that they needed information about the intruder's airspeed and vertical speed to solve the conflicts. Remarkably, the EVSD pilots also did not have this (explicit) information, but they also did not indicate a need for it. The VSD pilots found it also difficult to solve conflicts caused by intruder aircraft located behind the own aircraft. 
Therefore, they suggested to place the own aircraft in the center of the display (this was also indicated in earlier research ${ }^{13}$ ). EVSD pilots also wanted more information on the position of aircraft behind, but they did not regard that as a problem when solving the conflict. Further, pilots suggested to use the same aircraft symbols as used by TCAS and to include (aural) warnings for close conflicts.

\section{F. Discussion}

The visualization of the traffic and terrain constraints in combination with the internal performance constraints, has increased the traffic and terrain awareness of pilots and supported their decision-making.

There were no significant differences between the interfaces for performance/decision, but the results show a trend towards better performance and decisions for EVSD for traffic and mixed conflicts. There could be a number of reasons for the small differences between the results. First, it was difficult to define the best resolution maneuver for each scenario, because a resolution should be optimal in terms of both safety and efficiency. Safety and efficiency include many factors to optimize: distance, altitude, energy, fuel, time-of-arrival. The pilot task could have been more strictly formulated towards a predefined, optimal solution, but the goal was to investigate pilot behavior/decision-making in scenarios that reflect realistic situations, in which the pilot also has to make a trade-off between those factors. Second, dictating resolution strategies to pilots is against the principle of EID, because constraint-based interfaces present the functional constraints within which pilots should look for a solution. This knowledge challenged some pilots to find the most (flight path) efficient solution, while others were just satisfied with a safe resolution. After all, safety is the most important issue. The third reason for the small differences in performance could be that the design alternative was designed using the same principles.

Other measures for performance have been considered, such as flight path deviation. A large flight path deviation, however, does not necessarily mean that the conflict resolution is non-optimal. It could mean that the resolution is less efficient, but on the other hand more safe. Also, it was considered to record the reaction time of pilots. However, a short reaction time, for example, could mean a good understanding of the situation but also an emergency reaction because of a lack of information. It was observed that one pilot, operating with the VSD, had very short reaction times, but every time a conflict appeared (terrain or traffic) he started to climb immediately to avoid it.

Pilots operating with EVSD were more aware of the capabilities of the aircraft when avoiding the traffic and terrain obstacles. However, they had more difficulties in estimating the position and direction of the intruder aircraft. It is questionable whether this decrease in traffic awareness is justified if the pilot is perfectly able to obtain a resolution from the conflict geometry. Indeed, it was observed that the lack of an explicit speed indication of the intruder aircraft was not regarded awkward or vital to EVSD pilots, while most VSD pilots specifically asked for it.

To keep the number of variables limited, there was only one intruder aircraft present in each scenario. However, earlier research testing the same traffic constraints has indicated that multiple intruder aircraft did not lower SA significantly. ${ }^{13}$ Also to limit the number of variables, there were no scenarios with sudden system failures. Previous studies, however, indicated that constraint-based interfaces could be effective when pilots have to deal with unforeseen situations.

Pilot workload was lower for EVSD, although when a conflict was triggered, EVSD pilots needed more time to interpret the presented information. EVSD pilots, however, had a greater confidence about their choice, which resulted in lower mental and temporal workload. In order to maximize realism, pilots got multiple scenarios per measurement run (without freezing the simulation). A drawback of this method is that less (reliable) workload data was obtained, because the pilots could only complete a query after each run. Also, the fact that questions were asked (regarding SA) during flight, might have influenced pilot workload.

There were more terrain safety zone intrusions for EVSD. However, most pilots did not even notice that they violated the safety margin of $500 \mathrm{ft}$. After all, these maneuvers did not cause crashes and pilots are used to get visual and aural warnings when they approach terrain. Sometimes, pilots found themselves in situations where they had to use the constraint overlays for short-term conflict avoidance. Although the presented constraints still show the maneuver possibilities in these situations, pilots might not have the time to interpret the information. Then they should trust on alerting systems that provide an explicit conflict resolution. After all, constraint-based interfaces should prevent pilots to end up in such situations. Some pilots remarked that in short-term conflict situations they preferred an advisory escape maneuver instead of more SA. 
Although the experiment focused on professional airline operations, the EVSD might be more useful for general aviation, because those pilots are more used to maintain their own separation, the aircraft have less excess power and often fly without a predefined flight plan near terrain.

\section{Conclusions and Recommendations}

The experiment results show that pilots had a better SA with EVSD compared to the design alternative. Pilots were more aware of the capabilities of the aircraft when avoiding the traffic and terrain obstacles. However, a significant effect on performance was not measured. Pilots rated workload lower. There were more traffic protected zone intrusions with VSD, but more terrain intrusions with EVSD. No crashes were recorded.

For further development of the EVSD, it is recommended to explore other scenarios, such as scenarios with multiple intruders, changing aircraft configuration (flaps, speedbrakes, gear) and emergency/malfunction scenarios (limiting the performance of the aircraft). The traffic conflict detection algorithm should be improved to provide pilots better conflict information (in fact, the same algorithm as used for TCAS could be used). Also, intent information should be included to the display: flight plan information from the own aircraft and from other aircraft can be used to predict conflicts on their trajectories. This will reduce the number of false alarms shown on the EVSD. Finally, the effect of wind conditions on the performance envelope and solution space should be investigated since the EVSD relates the aircraft performance envelope, defined in the velocity domain, to distances relative to the ground.

\section{References}

${ }^{1}$ SESAR Consortium, "SESAR Definition Phase D3: The ATM Target Concept," Tech. Rep. DLM-0612-001-02-00, Eurocontrol, September 2007.

${ }^{2}$ RTCA, "Minimal Operational Performance Standards for Traffic Alert and Collision Avoidance System II (TCAS II)," Tech. Rep. DO-185B, Federal Aviation Authorities, June 2008.

${ }^{3}$ Honeywell, "Enhanced Ground Proximity Warning System: product description," Tech. rep., Honeywell Aerospace, http://www51.honeywell.com/aero/Products-Services/Avionics-Electronics/EGPWS-Home.html, retrieved: August, 2008.

${ }^{4}$ ACSS, T2CAS Product Description, Aviation Communication \& Surveillance Systems, June 2003.

${ }^{5}$ Pritchett, A. R., "Reviewing the Role of Cockpit Alerting Systems," Human Factors and Aerospace Safety, Vol. 1, No. 1, 2001, pp. 5-38.

${ }^{6}$ Bainbridge, L., "Ironies of Automation," New Technology and Human Error, 1987, edited by J. Rasmussen, John Wiley and Sons.

${ }^{7}$ Borst, C., Mulder, M., van Paassen, M. M., and Mulder, J. A., "An Ecological Approach to Support Pilot Terrain Awareness After Total Engine Failure," Journal of Aircraft, Vol. 45, No. 1, 2008, pp. 159-171.

${ }^{8}$ Borst, C., Suijkerbuijk, H. C. H., Mulder, M., and van Paassen, M. M., "Ecological Interface Design for Terrain Awareness," International Journal of Aviation Psychology, Vol. 16, No. 4, October 2006, pp. 375-400.

${ }^{9}$ Borst, C., Mulder, M., van Paassen, M. M., and Mulder, J. A., "Theoretical Foundations of an Ecological Synthetic Vision Display," AIAA Guidance, Navigation, and Control Conference, No. AIAA-2009-5982-357, Chicago, Illinois, August 2009.

${ }^{10}$ Borst, C., Mulder, M., van Paassen, M. M., and Mulder, J. A., "Experimental Evaluation of an Ecological Synthetic Vision Display," AIAA Guidance, Navigation, and Control Conference, No. AIAA-2009-5983-909, Chicago, Illinois, August 2009.

${ }^{11}$ van Dam, S. B. J., Mulder, M., and van Paassen, M. M., "Ecological Interface Design of Conflict Support in Flexible Use Airspace," Proceedings of the AIAA Guidance, Navigation and Control Conference and Exhibit, August 2005.

12 van Dam, S. B. J., Mulder, M., and van Paassen, M. M., "Including Turn Dynamics and Intruder Intent Information on An Airborne Self-Separation Assistance Display," Proceedings of the IEEE Conference on Systems, Man, ES Cybernetics (IEEE-SMC), Montreal, Candada, October 8 - 11, 2007, pp. 1445-1454.

${ }^{13}$ Heylen, F. M., S. B. J. van Dam, M. M., and van Paassen, M. M., "Design and Evaluation of a Vertical Separation Assitance Display," AIAA Guidance, Navigation, and Control Conference and Exhibit, No. AIAA-2008-6969, August 2008.

${ }^{14}$ Suijkerbuijk, H. C. H., Borst, C., Mulder, M., and van Paassen, M. M., "Development and Experimental Evaluation of a Performance-Based Vertical Situation Display," No. AIAA-2005-5961-466 in AIAA Guidance, Navigation, and Control Conference and Exhibit, San Francisco, California, August 2005.

${ }^{15}$ van der Linden, C. A. A. M., "DASMAT - Delft University Aircraft Simulation Model and Analysis Tool, a Matlab/Simulink Envrionment for Flight Dynamics and Control Analysis," Tech. Rep. LR-781, August 1996.

${ }^{16}$ Field, A., Discovering Statistics Using SPSS, SAGE Publications Ltd., 2nd ed., 2005. 